\title{
Effects of Different Working Modes of Ultrasound on Structural Characteristics of Zein and ACE Inhibitory Activity of Hydrolysates
}

\author{
Xiaofeng Ren, ${ }^{1,2}$ Xi Zhang, ${ }^{1}$ Qiufang Liang, ${ }^{1}$ Ting Hou, ${ }^{1}$ and Huiji Zhou ${ }^{1}$ \\ ${ }^{1}$ School of Food and Biological Engineering, Jiangsu University, 301 Xuefu Road, Zhenjiang, Jiangsu 212013, China \\ ${ }^{2}$ Jiangsu Provincial Key Laboratory for Physical Processing of Agricultural Products, Zhenjiang, Jiangsu 212013, China \\ Correspondence should be addressed to Xiaofeng Ren; renxiaofeng@ujs.edu.cn
}

Received 31 July 2017; Revised 9 November 2017; Accepted 21 November 2017; Published 13 December 2017

Academic Editor: Giuseppe Zeppa

Copyright ( $\odot 2017$ Xiaofeng Ren et al. This is an open access article distributed under the Creative Commons Attribution License, which permits unrestricted use, distribution, and reproduction in any medium, provided the original work is properly cited.

Ultrasound was used as a new technology to pretreat protein prior to proteolysis to improve enzymolysis efficiency. The effects of different working modes of ultrasound on the angiotensin I-converting enzyme (ACE) inhibitory activity of zein hydrolysates and the structural characteristics of zein were investigated. The solubility, surface hydrophobicity $\left(H_{0}\right)$, ultraviolet-visible (UV-Vis) spectra, intrinsic fluorescence spectra, and circular dichroism (CD) spectra of zein pretreated with ultrasound were determined. All ultrasound pretreatments significantly improved the ACE inhibitory activity of zein hydrolysates $(p<0.05)$. The highest ACE inhibitory activity, representing an increase of $99.21 \%$ over the control, was obtained with dual sweeping frequency ultrasound of $33 \pm 2$ and $68 \pm 2 \mathrm{kHz}$. The effects of single sweeping frequency and dual fixed frequency ultrasound were stronger than those of single fixed frequency ultrasound for improving the ACE inhibitory activity of zein. Structural changes in zein were induced by ultrasound, as confirmed by changes in the solubility, $H_{0}$, UV-Vis spectra, intrinsic fluorescence spectra, and CD spectra of zein, and these were consistent with the corresponding ACE inhibitory activities of zein hydrolysates. Thus, ultrasound working mode and frequency have significant effects on the structure of zein and the ACE inhibitory activity of zein hydrolysates.

\section{Introduction}

Corn gluten meal, a main byproduct from the corn wetmilling process, contains $60-71 \%(\mathrm{w} / \mathrm{w})$ protein. The major protein fractions in corn gluten meal are zein and glutelin, accounting for $68 \%$ and $28 \%$, respectively, of the total protein weight [1]. At present, corn gluten meal is mainly used as a foodstuff or disposed of, largely due to the water insolubility of zein and the severe imbalance of amino acids that compose it [2]. Zein belongs to the class of prolamins with a molecular weight of apparently $24 \mathrm{kDa}$ and $27 \mathrm{kDa}(\alpha-$ zein) and $17 \mathrm{kDa}(\beta$-zein) and consists of mostly hydrophobic nonpolar amino acids that are buried inside the molecule [3]. It has been reported that the bioavailability of its proteins could be improved significantly via enzymatic modification [4]. However, the water insolubility of zein and its extensive intermolecular interactions create a barrier, making it difficult for the enzyme to attack the protein bonds and leading to long processing times and low enzyme utilization rates and protein conversion rates. Therefore, innovative technologies to overcome these shortcomings are in high demand.

Low-frequency $(20-100 \mathrm{kHz})$ ultrasound treatment, which has been widely used in the extraction of target compounds, production of bioactive peptides, and hydrolysis of proteins [5], has attracted increasing attention in the food processing industry. Owing to its thermal, cavitation, and mechanical effects, ultrasound can increase mass transfer, enhance the extrusion of media molecules, and change media density, function, and structure. Especially for acoustic cavitation, the collapse of cavitation bubbles can produce violent physical forces (e.g., microjets, shear forces, and shock waves) and free radicals [6]. Ultrasound is considered the fundamental process that causes the most sonochemical reactions in liquids [7]. Acoustic mode and ultrasonic frequency are two major factors that affect the production and intensity of liquid cavitation and influence 


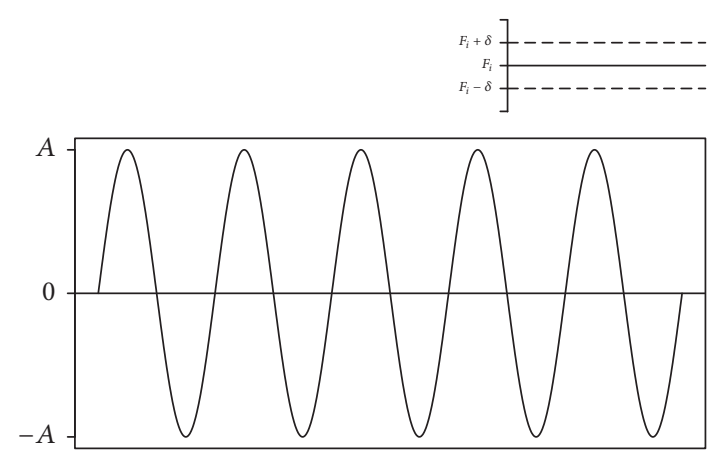

(a)

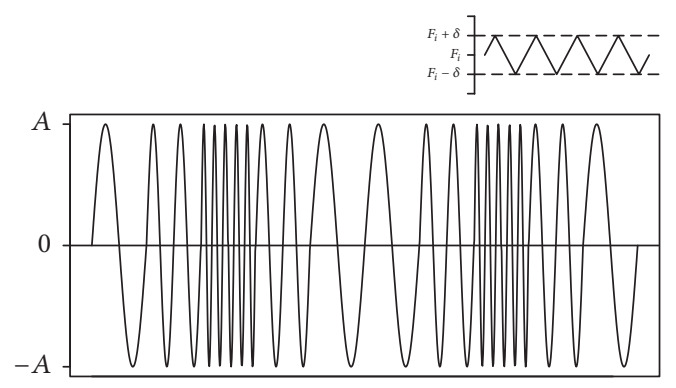

(c)

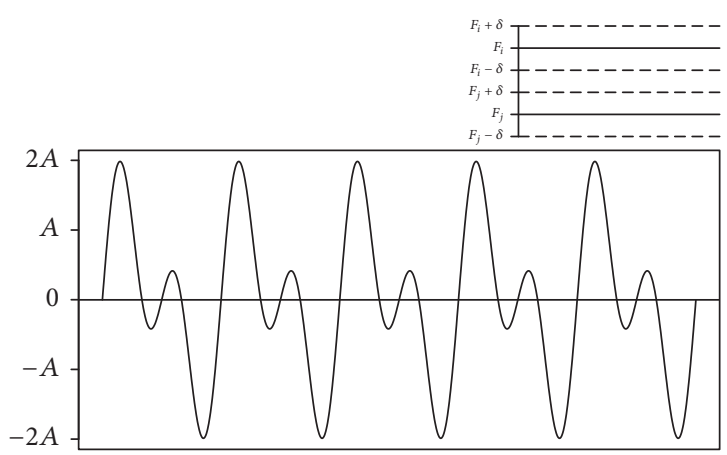

(b)

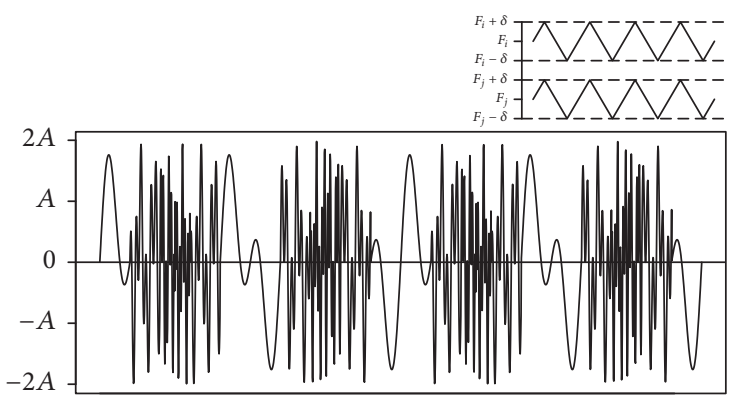

(d)

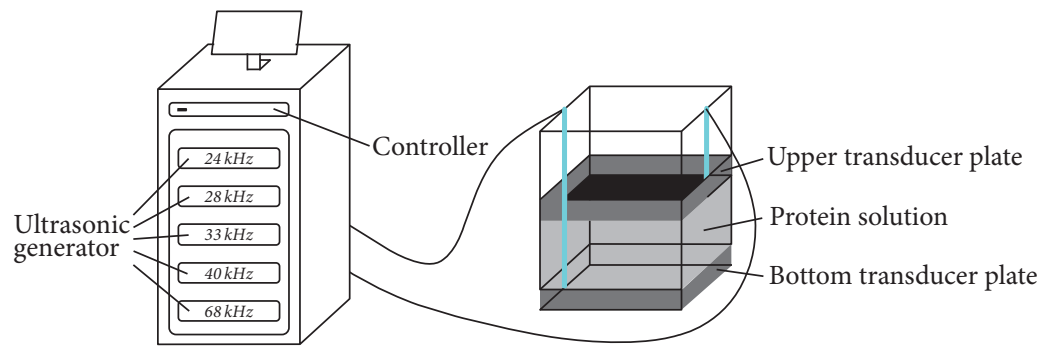

(e)

FigURE 1: Ultrasonic patterns of single fixed frequency ultrasound (a), dual fixed frequency ultrasound (b), single sweeping frequency ultrasound (c), and dual sweeping frequency ultrasound (d). Equipment used for fixed and sweeping frequency ultrasound (e).

the ultrasound treatment effect. Qu et al. [8] found that a single sweeping frequency ultrasound pretreatment at $24 \pm 2 \mathrm{kHz}$ can increase the enzymolytic efficiency and angiotensin-converting enzyme (ACE) inhibitory activity of wheat germ protein hydrolysates. Jin et al. $[9,10]$ reported that dual sweeping frequency ultrasound pretreatment at $28 \pm 2 / 68 \pm 2 \mathrm{kHz}$ can change the surface microstructure and chemical structure of proteins. Jia et al. [11] demonstrated that ultrasound with a single fixed frequency of $24 \mathrm{kHz}$ resulted in increases of $21.0-40.7 \%$ in the ACE inhibitory activity of wheat germ protein hydrolysates. Ultrasound pretreatment can significantly change the structure of proteins and improve the functionality of protein hydrolysates. There have also been several studies focused on pretreatments with simple ultrasound modes of one or several frequencies on different raw proteins. However, there has been no systematic research conducted on the effect of different ultrasound frequencies and working mode pretreatments on raw materials.

Our team has researched the application of different working modes of ultrasound technology in food physical processing for many years. The equipment for fixed frequency and sweeping frequency ultrasonic baths has been well developed, consisting of upper and lower plates (Figure 1(e)), each of which can deliver its own frequency separately. In general, there are four working modes of ultrasound with different frequencies induced by this equipment: single fixed frequency $\left(f_{i} \mathrm{kHz}\right)$ ultrasound (Figure 1(a)), dual fixed frequency $\left(f_{i}\right.$ and $f_{j} \mathrm{kHz}$ ) ultrasound (Figure 1(b)), single sweeping frequency $\left(f_{i} \pm \delta \mathrm{kHz}\right)$ ultrasound (Figure $1(\mathrm{c})$ ), and dual sweeping frequency $\left(f_{i} \pm \delta\right.$ and $\left.f j \pm \delta \mathrm{kHz}\right)$ ultrasound (Figure $1(\mathrm{~d})$ ). On the basis of an earlier report, we previously investigated the effect of single-frequency ultrasound treatment on enzymatic preparations of ACE inhibitory peptides from zein [12]. However, there is limited information regarding the effect of dual-frequency ultrasonic treatment on zein. Therefore, the objective of this research was to (1) compare the effects of pretreatment with the four working modes of ultrasound at two frequencies on the degree of hydrolysis $(\mathrm{DH})$ of zein and the ACE inhibitory activity of 
zein hydrolysates and (2) compare resulting changes in the microstructure of zein.

\section{Materials and Methods}

2.1. Materials. Corn gluten meal with $56-60 \%$ protein content was obtained from Fenda Starch Co. Ltd. (Jiangsu, China). Zein with $90.23 \%$ protein purity was extracted according to the methods of a previous study [13]. Alcalase with an activity of $132,507 \mathrm{U} / \mathrm{ml}$ was purchased from Novozymes Biotechnology Co. Ltd. (Shanghai, China). ACE was extracted from pig lungs, and its activity was assessed using Hippuryl-His-Leu (Sigma-Aldrich Trading Co. Ltd., Shanghai, China) as a substrate. All other reagents were of analytical grade.

2.2. Ultrasound Pretreatment of Zein. An aliquot of zein suspension $(200 \mathrm{~mL}, 2 \mathrm{~g}$ zein) was sealed in a high-pressureresistant bag and treated in an ultrasound bath (internal dimensions $362 \mathrm{~mm} \times 294 \mathrm{~mm} \times 502 \mathrm{~mm}$; Shangjia Biotechnology Co., Wuxi, Jiangsu, China) with $5.4 \mathrm{~L}$ of water. The two frequencies tested were 33 and $68 \mathrm{kHz}$. The four working modes of operation included single fixed frequency $(33 \mathrm{kHz}$ or $68 \mathrm{kHz}$ ) ultrasound, dual fixed frequency ( 33 and $68 \mathrm{kHz}$ ) ultrasound, single sweeping frequency $(33 \pm 2 \mathrm{kHz}$ or $68 \pm$ $2 \mathrm{kHz}$ ) ultrasound, and dual sweeping frequency (33 \pm $2 \& 68 \pm 2 \mathrm{kHz}$ ) ultrasound. The other pretreatment parameters were as follows: sweeping cycle, $500 \mathrm{~s}$; power, $600 \mathrm{~W}$; pulsed on/off time, $10 \mathrm{~s} / 3 \mathrm{~s}$; and sonication time, $40 \mathrm{~min}$. Control samples were prepared using a magnetic stirrer rather than ultrasound treatment, with other conditions remaining unchanged.

To study the effect of ultrasound on the structural characteristics of zein, precipitation was performed by centrifugation at 2,500 $\mathrm{g}$ for $10 \mathrm{~min}$ and lyophilization for further analysis.

2.3. Enzymatic Hydrolysis of Zein. Before enzymatic hydrolysis, the zein suspension was adjusted to $\mathrm{pH} 8.0$ and incubated in a water bath at $50^{\circ} \mathrm{C}$ for $10 \mathrm{~min}$. Then, Alcalase $(E / S=$ $3500 \mathrm{U} / \mathrm{g}$ ) was added to start the reaction, and the $\mathrm{pH}$ was maintained at 8.0 by continuously adding $1 \mathrm{M} \mathrm{NaOH}$. After enzymatic hydrolysis for $40 \mathrm{~min}$, the mixture was adjusted to $\mathrm{pH} 7.0$ and boiled for $10 \mathrm{~min}$ to inactivate the enzyme. Trichloroacetic acid $(6 \% \mathrm{v} / \mathrm{v})$ was added to the mixture at a ratio of $1: 1$ to precipitate the protein. Finally, the hydrolysates were cooled and centrifuged at $5000 \mathrm{~g}$ for $15 \mathrm{~min}$, and the supernatant was acquired for further analysis.

\subsection{Determination of Enzymatic Hydrolysis of Zein}

2.4.1. Degree of Hydrolysis. The DH of zein was determined using the $\mathrm{pH}$-state method [14] and was calculated using

$$
\mathrm{DH}(\%)=\frac{h}{h_{\mathrm{tot}}}=\frac{N_{b} \times B \times 100}{\alpha \times M_{p} \times h_{\mathrm{tot}}},
$$

where $B$ is the $\mathrm{NaOH}$ volume consumed $(\mathrm{mL}), N_{b}$ is the normality of the $\mathrm{NaOH}(\mathrm{mmol} / \mathrm{mL}), M_{p}$ is the protein weight (g), $\alpha$ is the average dissociation degree of $\alpha-\mathrm{NH}_{2}$ in substrate
( 0.985 for zein), and $h_{\text {tot }}$ is the total number of peptide bonds in the protein substrate $(7.35 \mathrm{mmol} / \mathrm{g}$ for zein).

2.4.2. Measurement of ACE Inhibitory Activity. The ACE inhibitory activity of the zein hydrolysates was measured using a previously reported method with slight modifications [15]. Briefly, $45 \mu \mathrm{L}$ of substrate borate buffer (1.0 mM FAPGG, $80 \mathrm{mM}$ HEPES, $0.1 \mathrm{M}$ borate buffer, and $300 \mathrm{mM} \mathrm{NaCl}$; $\mathrm{pH}$ 8.3) was mixed with $40 \mu \mathrm{L}$ of sample in a microliter plate well. The reaction was initiated by adding $20 \mu \mathrm{L}$ of ACE $(0.1 \mathrm{U} / \mathrm{mL})$. After reacting for $30 \mathrm{~min}$ at $37^{\circ} \mathrm{C}$, the absorbance of the reaction mixture was immediately monitored at $340 \mathrm{~nm}$. The ACE inhibitory activity was calculated as follows:

$$
I=\frac{\left(A_{C}-A_{S}\right)}{\left(A_{C}-A_{B}\right)} \times 100,
$$

where $I$ is the ACE inhibitory activity (\%), $A_{C}$ is the absorbance of the control group without protein hydrolysates, $A_{B}$ is the absorbance of the blank group without ACE, and $A_{S}$ is the absorbance of the sample group with ACE and protein hydrolysates.

\subsection{Determination of Physicochemical Properties and Structure of Zein}

2.5.1. Solubility Measurement. Bovine serum albumin was chosen as a standard, and the crude protein content of the supernatant after ultrasound treatment was determined according to the Lowry method [16]. Solubility was expressed as the percentage of protein in the supernatant compared with the total amount of primary protein.

2.5.2. Surface Hydrophobicity $\left(H_{0}\right)$ Measurement. The surface hydrophobicity of zein dispersions was determined using the method described by Kato and Nakai [11, 17] with slight modifications using 1-anilino-8-naphthalene-sulfonate as a fluorescent probe (ANS). The ultrasound-pretreated and control samples were dissolved in $0.01 \mathrm{M}$ phosphate buffer saline (PBS) to $0.5 \mathrm{mg} / \mathrm{mL}$. Then, $50 \mu \mathrm{L}$ of ANS was added to the $5 \mathrm{~mL}$ of zein supernatant and mixed at $25^{\circ} \mathrm{C}$. Relative fluorescence intensity was monitored immediately using a Cary Eclipse spectrophotometer at an excitation wavelength of $700 \mathrm{~nm}$ (slit $5.0 \mathrm{~nm}$ ), emission wavelength of $360-750 \mathrm{~nm}$, and scanning speed of $5 \mathrm{~nm} / \mathrm{s}$. The surface hydrophobicity was expressed as the relative fluorescence intensity at a protein concentration of $0.5 \mathrm{mg} / \mathrm{mL}$.

2.5.3. Ultraviolet-Visible (UV-Vis) Spectroscopy. Freeze-dried protein samples were dissolved in $60 \%(\mathrm{v} / \mathrm{v})$ ethanol to $0.2 \mathrm{mg} / \mathrm{mL}$. They were then scanned, and ultravioletvisible spectra were recorded in the wavelength range of 200-400 nm using a Varian Cary 100 UV-Vis spectrophotometer (Varian Inc., Palo Alto, USA) at $25^{\circ} \mathrm{C}$ with a $60 \mathrm{~nm} / \mathrm{min}$ scan rate and a $2.0 \mathrm{~nm}$ bandwidth, in a quartz cell with a path length of $1.0 \mathrm{~cm}$.

2.5.4. Measurement of Intrinsic Fluorescence Spectrum. The intrinsic fluorescence emission spectrum of zein $(0.2 \mathrm{mg} / \mathrm{mL}$ 


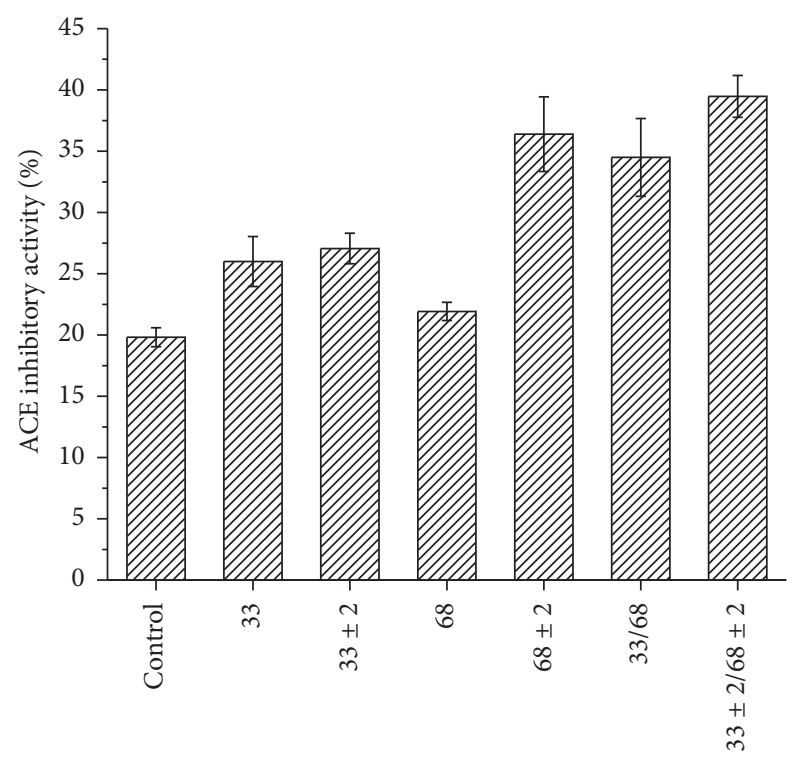

Frequency $(\mathrm{kHz})$

FIGURE 2: Effects of pretreatment with ultrasound bath with four different working modes and two frequencies on the ACE inhibitory activity of zein hydrolysates.

in $60 \%$ ethanol) was measured at $25^{\circ} \mathrm{C}$ using a Cary Eclipse fluorescence spectrophotometer (Varian Inc., Palo Alto, USA) equipped with a cell with a path length of $1 \mathrm{~cm}$, using an excitation wavelength of $350 \mathrm{~nm}$ (slit $=5 \mathrm{~nm}$ ), emission wavelength of $360-750 \mathrm{~nm}$ (slit $=5 \mathrm{~nm}$ ), and scanning speed of $20 \mathrm{~nm} / \mathrm{s}$.

2.5.5. Circular Dichroism (CD) Spectroscopy. The CD spectra of zein $(0.2 \mathrm{mg} / \mathrm{mL}$ in $60 \%$ ethanol) were assessed at a wavelength range of $190-250 \mathrm{~nm}$ with a Jasco J-715 CD spectropolarimeter (Jasco Corp., Tokyo, Japan) using a quartz cuvette with a $10-\mathrm{mm}$ optical path length under nitrogen flux at a temperature of $25^{\circ} \mathrm{C}$, scanning rate of $50 \mathrm{~nm} / \mathrm{min}$, and bandwidth of $1.0 \mathrm{~nm}$. The content of the protein secondary structure was calculated from the far-UV CD spectra using the DICHROWEB procedure [18-22].

2.6. Statistical Analysis. All experiments were performed at least in duplicate, and the results are presented as the mean \pm standard deviation. Differences among treatments were determined by analysis of variance (ANOVA), and $p<0.05$ was considered significant. All graphs were generated and calculations were performed using OriginPro8.5 and SPSS 18.0.

\section{Results and Discussion}

3.1. Effect of Ultrasound Pretreatment on ACE Inhibitory Activity of Zein Hydrolysates. The ACE inhibitory activity of zein hydrolysates pretreated with ultrasound was higher than that of the control, to varying degrees, and is presented in Figure 2. At a single fixed frequency of $68 \mathrm{kHz}$, the ACE inhibitory activity was only increased by $10.62 \%$ over that of the control, representing a smaller increase than that with a single fixed frequency of $33 \mathrm{kHz}$ ( $31.18 \%$ increase). This finding that a

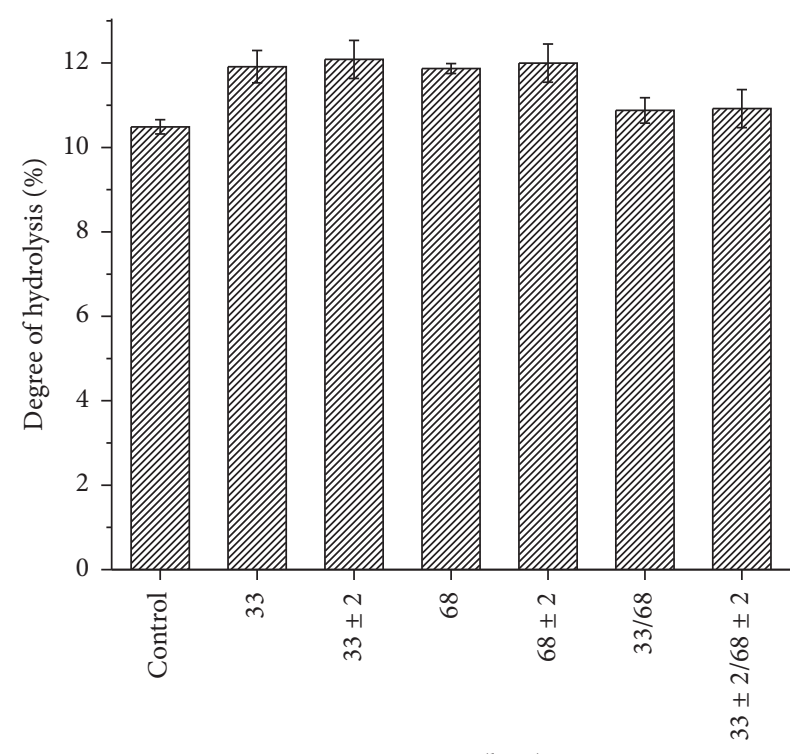

Frequency $(\mathrm{kHz})$

FIGURE 3: Effects of pretreatment with ultrasound bath with four different working modes and two frequencies on the degree of hydrolysis of zein.

lower frequency induced a higher ACE inhibitory activity is consistent with a report on defatted wheat germ proteins [1], which attributed this decrease to controlling the attenuation of ultrasound at high frequencies, resulting in lower energy loss [23]. In the case of ultrasound pretreatment with a single sweeping frequency of $68 \pm 2 \mathrm{kHz}$, we found that the ACE inhibitory activity was higher than that for the ultrasound treatment with a dual fixed frequency of 33 and $68 \mathrm{kHz}$. This might be due to the resonance frequency of ultrasound. Notably, with a dual sweeping frequency of $33 \pm 2$ and $68 \pm$ $2 \mathrm{kHz}$, the ACE inhibitory activity was significantly increased, reaching a maximum (99.21\% increase). Compared with the dual fixed frequency, the dual sweeping frequency was more conducive to increasing the ACE inhibitory activity of zein hydrolysates. Arzeni et al. [24] reported that dual-frequency ultrasound can significantly increase the cavitation yield compared with that by two individual ultrasonic irradiation types. This higher cavitation yield is due to increased transfer of the input electrical energy and an increased resonance effect, which contribute to bubble growth and yield the maximum bubble size [25]. Furthermore, the dual sweeping frequency ultrasound contributed to unfolding of the molecular structure of zein and hydrolysis of zein-releasing peptides with high ACE inhibitory activity.

3.2. Effect of Ultrasound Pretreatment on Degree of Hydrolysis (DH) of Zein. Figure 3 shows the $\mathrm{DH}$ values for control and ultrasound-assisted enzymolysis of zein. The 33, $33 \pm 2$, 68 , and $68 \pm 2 \mathrm{kHz}$ ultrasound pretreatments significantly improved the DH of zein by $13.13-15.22 \%$, which may reflect changes in the molecular conformation of the protein. As zein has a three-dimensional spatial structure and predominantly hydrophobic nonpolar amino acid composition, Alcalase 


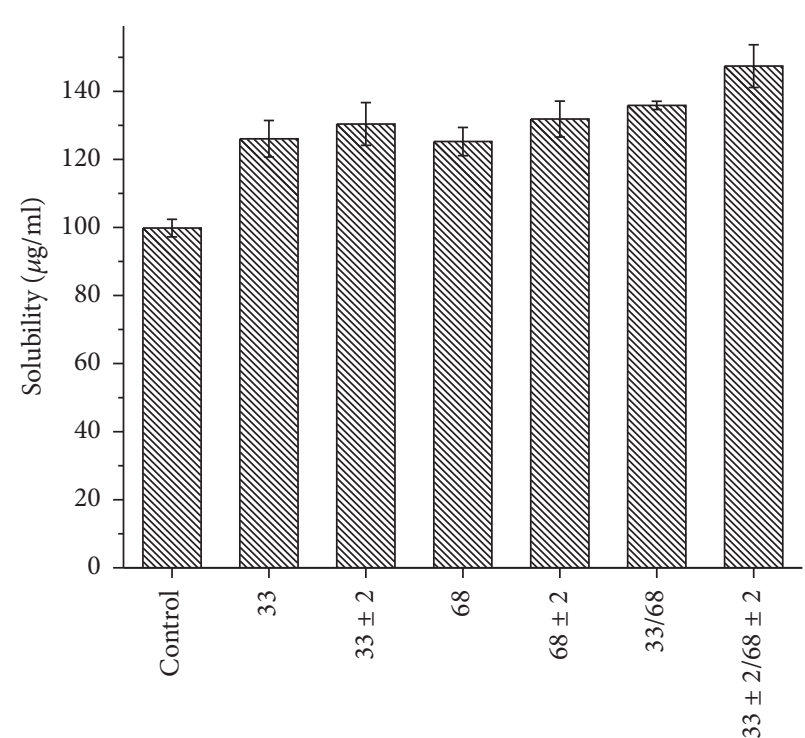

Frequency $(\mathrm{kHz})$

(a)

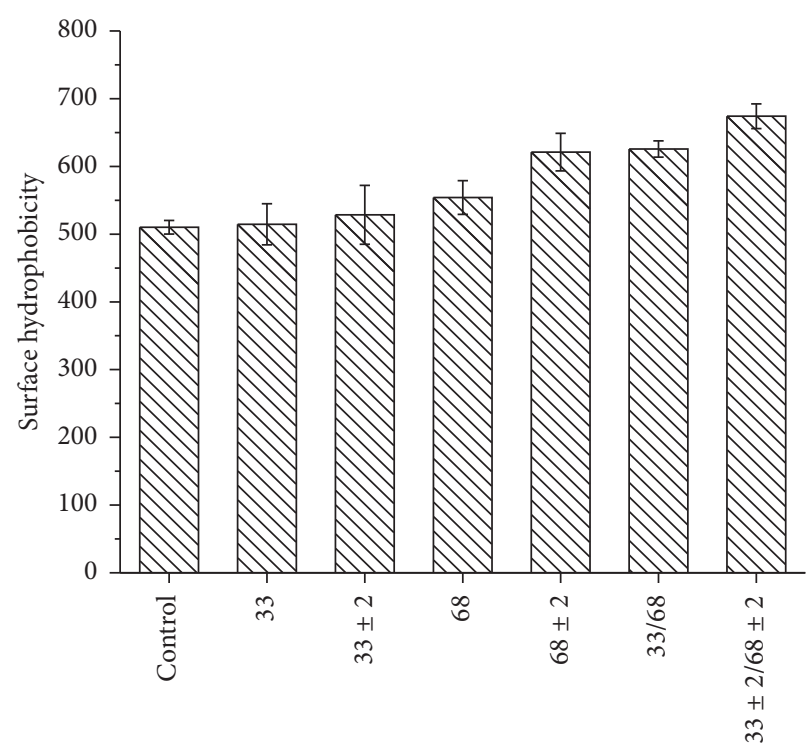

Frequency $(\mathrm{kHz})$

(b)

FIGURE 4: Effects of pretreatment with ultrasound bath with four different working modes and two frequencies on the solubility (a) and surface hydrophobicity (b) of zein.

did not hydrolyze zein effectively [3]. After treatment with ultrasound, however, the protein macromolecules became more loosely arranged, with peptide bonds previously buried inside the protein being exposed $[9,26]$. This increases the probability of the enzyme coming into contact with the active site of the protein. In contrast, pretreatment using dualfrequency ultrasound had little effect on the $\mathrm{DH}$ of zein, although the ACE inhibitory activity of the zein hydrolysates was considerably enhanced under the same conditions. This phenomenon is consistent with that reported by Sun et al. [27] and Zhou et al. [1], who found that some ultrasound pretreatments have little effect on protein DH but significantly increase the ACE inhibitory activity of protein hydrolysates. This suggests that there is no direct relationship between increases in ACE inhibitory activity and the DH of zein.

\subsection{Effect of Ultrasound Pretreatment on Structural Characteristics of Zein}

3.3.1. Effect of Ultrasound Pretreatment on Solubility of Zein. Solubility is the most effective way to measure the degree of protein denaturation and aggregation in solution, and it is a good indicator of protein function [28]. After ultrasound treatment, the solubility of zein was significantly increased by 20.81-32.28\% compared with that of the control (Figure 4(a)). These results indicate that ultrasound enhances the unfolding of protein molecules, exposure of hydrophilic amino acids, and interaction between proteins and water molecules. This result is consistent with similar findings for ultrasoundpretreated proteins, such as black bean protein isolate [29], soy protein isolate [30], and milk protein concentrate [31]. The different working modes of ultrasound greatly changed the solubility, with the highest solubility observed in the sample that was treated with dual sweeping frequency ultrasound at $33 \pm 2$ and $68 \pm 2 \mathrm{kHz}$. Thus, treatment using dual sweeping frequency ultrasound is more efficient at increasing the solubility of zein than the other working modes of ultrasound.

3.3.2. Effects of Ultrasound Pretreatment on the Hydrophobicity $\left(H_{0}\right)$ of Zein. $H_{0}$ of a protein is one of the structural features used to evaluate changes in protein conformation that affect functional properties and stability [17]. Figure 4(b) shows that ultrasound treatment increased $H_{0}$ of zein to varying degrees. Comparing the $H_{0}$ values of zein when sonicated at the two frequencies, single-frequency ultrasound treatments of $33 \mathrm{kHz}$ and $33 \pm 2 \mathrm{kHz}$ resulted in only small increases in $H_{0}$, indicating that $H_{0}$ increased with increasing ultrasonic frequency $(33 \mathrm{kHz}$ versus $68 \mathrm{kHz})$. For zein treated with ultrasound of different working modes $(68 \mathrm{kHz}$ versus $68 \pm 2 \mathrm{kHz}$ ), the effect on $H_{0}$ was stronger with sweeping ultrasound treatment than with fixed frequency ultrasound. In addition, the largest increase in $H_{0}$ was observed with treatment with dual sweeping frequency ultrasound at $33 \pm 2$ and $68 \pm 2 \mathrm{kHz}$. The results for the ACE inhibitory activity of the zein hydrolysates and the solubility of zein pretreated by dual sweeping frequency ultrasound at $33 \pm 2$ and $68 \pm$ $2 \mathrm{kHz}$ are therefore consistent with the results for $H_{0}$. These findings suggest that the differences in the $H_{0}$ of zein were due to differences in the working mode and frequency of the ultrasound, which may change the structure of the zein macromolecule and result in more or fewer hydrophobic groups on the inside of the protein molecule being exposed. trum of Zein. Figure 5 shows the typical UV-Vis spectra 


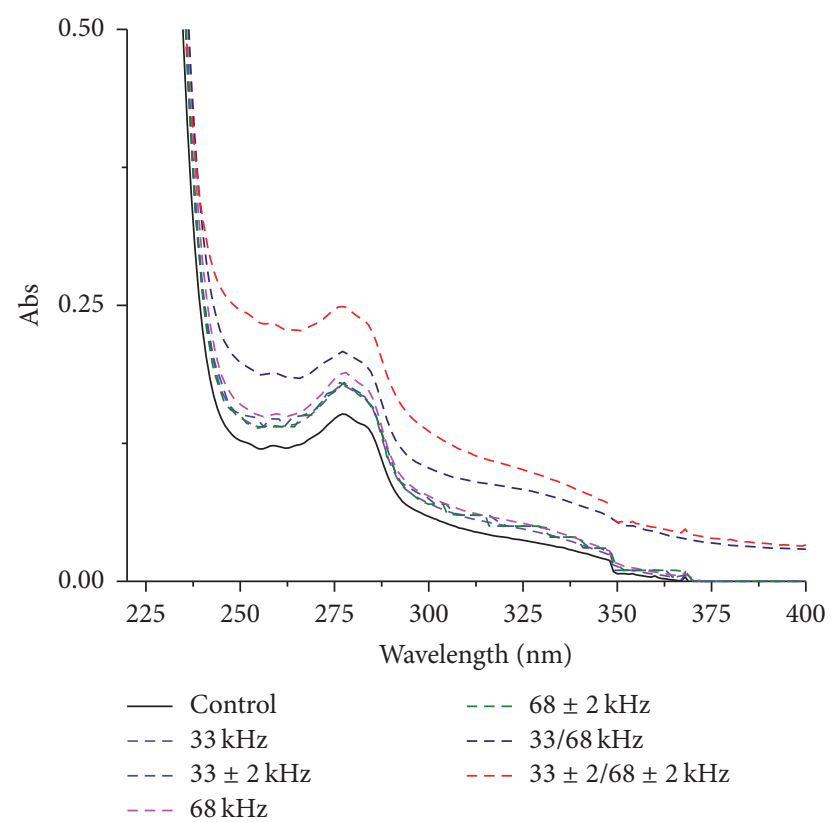

FIGURE 5: Effects of pretreatment with ultrasound bath with four different working modes and two frequencies on the ultravioletvisible spectra of zein.

of zein after ultrasonic treatment using the four working modes and the two frequencies. Tyrosine, tryptophan, and phenylalanine have characteristic absorptions of $275 \mathrm{~nm}$, $279 \mathrm{~nm}$, and $257 \mathrm{~nm}$, respectively [32]. These hydrophobic amino acids usually prefer to be buried in hydrophobic protein cores. As shown by the spectra, the absorbance intensity of zein macromolecules was increased by ultrasound treatment, indicating that ultrasonic treatment contributed to the exposure of hydrophobic amino acids. This finding is similar to that of Jin et al. [9] in corn gluten meal. The effect of dual sweeping frequency ultrasound pretreatment on the absorbance of zein was much stronger than that of the other ultrasound treatments, possibly because the combination of frequencies for sweeping frequency ultrasound has a resonance effect.

\subsubsection{Effect of Ultrasound Pretreatment on Intrinsic Fluores-} cence Spectrum of Zein. Because the intrinsic fluorescence of aromatic amino acid (tryptophan, tyrosine, and phenylalanine) residues is sensitive to the polarity of the microenvironment during transformation, intrinsic fluorescence has been shown to be an efficient method of determining the tertiary structure transition for soluble proteins. Zein in aqueous ethanol was excited at $350 \mathrm{~nm}$ and scanned with an emission wavelength of $360-750 \mathrm{~nm}$. As shown in Figure 6, the emission fluorescence intensity reached maximum values at wavelengths of 420 and $700 \mathrm{~nm}$. Compared with that of the control, the relative proportions of the fluorescence peak areas (at $420 \mathrm{~nm}$ ) of zein after pretreatment with ultrasound increased, agreeing with findings of $\mathrm{Li}$ et al. Moreover, the effect of dual sweeping frequency ultrasound on the absorbance intensity was significantly stronger than that

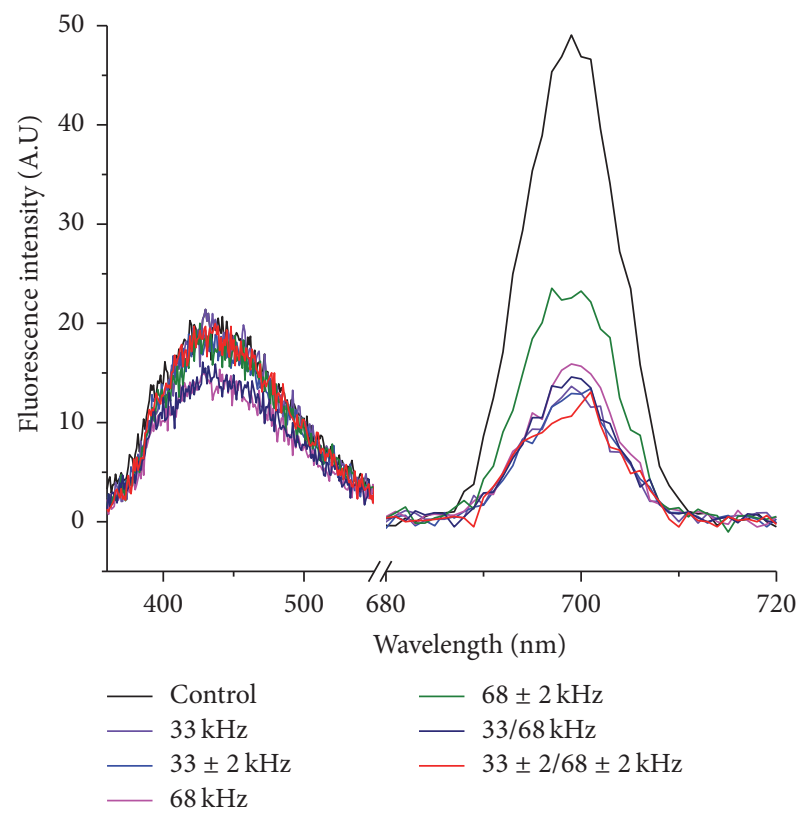

FIGURE 6: Effects of pretreatment with ultrasound bath with four different working modes and two frequencies on the intrinsic fluorescence spectra of zein.

of the other ultrasound pretreatments. The above results indicate that ultrasound pretreatment can alter the protein conformation and disrupt protein bonds, leading to increased exposure of buried hydrophobic groups.

3.3.5. Effects of Ultrasound Pretreatment on the Circular Dichroism (CD) of Zein. To obtain further information on the changes in protein structure induced by ultrasound, the effects of ultrasound pretreatment at different frequencies and working modes on the secondary and tertiary structures of zein were investigated. $\alpha$-Helix, $\beta$-sheet, $\beta$-turn, and random coil structures, each, give rise to a $C D$ spectrum of characteristic shape and magnitude. The CD spectra of zein are shown in Figure 7. Ultrasound pretreatment clearly increased the intensity of the positive peak, whereas the opposite was true for the negative peak. These changes reflect an increase in the disorder of the protein molecules. To quantify the changes in zein structure, the DICHROWEB procedure was used to calculate the $\alpha$-helix, $\beta$-sheet, $\beta$ turn, and random coil contents (Table 1). There was a slight increase in the $\alpha$-helix content of zein treated by ultrasound. However, Li et al. reported contradictory findings showing that sonication decreased the $\alpha$-helix content of rice protein. In contrast, Jayani et al. found that ultrasound resulted in a $10 \%$ increase in the $\alpha$-helix content of whey protein, which is in agreement with the present results. Therefore, the difference between the present results and those of Li et al. obtained for rice protein might reflect differences in the native protein and ultrasound conditions. Compared with the control, ultrasound treatment induced considerable increases in the $\beta$-sheet, $\beta$-turn, and random coil contents of zein, which is consistent with findings reported by Jin et al. [9]. This phenomenon may be due to the formation of localized 
TABLE 1: Effects of pretreatment with ultrasound bath with four different working modes and two frequencies on the secondary structure content (\%) of zein.

\begin{tabular}{lccc}
\hline Treatment & $\alpha$-Helix & $\beta$-Sheet and $\beta$-Turn & Random coil \\
\hline Control & 79.6 & 7.8 & 7.3 \\
$33 \mathrm{kHz}$ & $83.7(4.90 \%)$ & $13.2(40.90 \%)$ & $11.1(34.23 \%)$ \\
$33 \pm 2 \mathrm{kHz}$ & $83.4(4.55 \%)$ & $13.2(40.90 \%)$ & $18.7(60.96 \%)$ \\
$68 \mathrm{kHz}$ & $80.6(1.24 \%)$ & $7.2(-8.33 \%)$ & $7.0(-4.29 \%)$ \\
$68 \pm 2 \mathrm{kHz}$ & $84.5(5.79 \%)$ & $12.4(37.09 \%)$ & $18.4(60.32 \%)$ \\
$33 \& 68 \mathrm{kHz}$ & $84.5(5.79 \%)$ & $12.1(35.53 \%)$ & $18.7(60.96 \%)$ \\
$33 \pm 2 \& 68 \pm 2 \mathrm{kHz}$ & $81.8(2.69 \%)$ & $9.7(19.59 \%)$ & $17.1(57.30 \%)$ \\
\hline
\end{tabular}

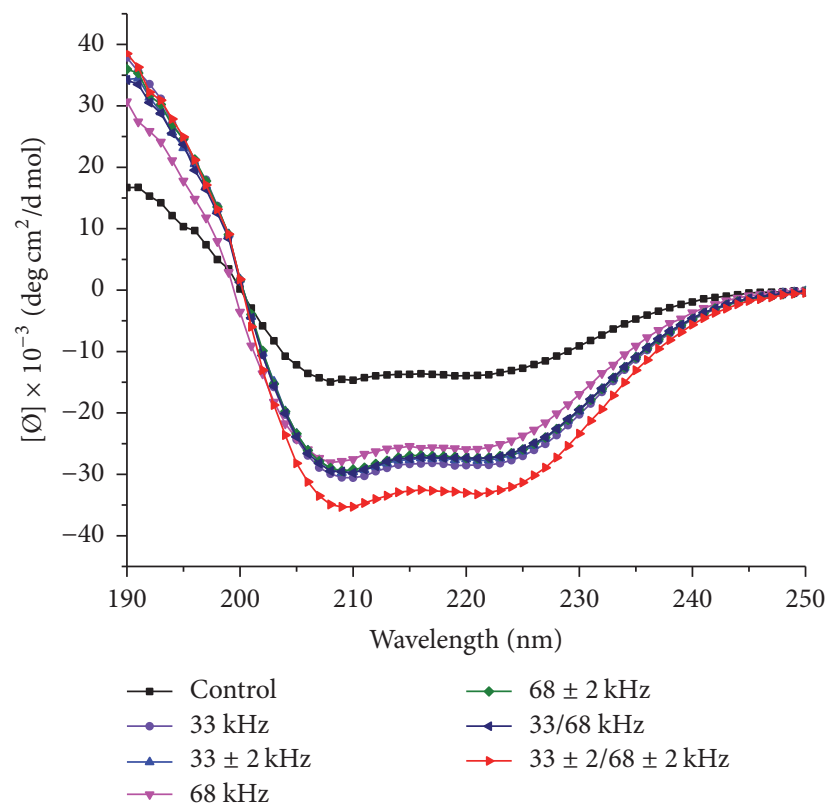

FIGURE 7: Effects of pretreatment with ultrasound bath with four different working modes and two frequencies on the circular dichroism spectra of zein.

hot spots upon the collapse of bubbles, shear forces created by shock, and microstreaming waves induced by ultrasound, which expose amino acid residues buried in the hydrophobic core of the zein protein and disrupt and rearrange interactions between protein molecule chains. However, with ultrasound treatment at a frequency of $68 \mathrm{kHz}$, the $\beta$-sheet, $\beta$ turn, and random coil contents decreased, which may suggest protein molecular aggregation. This indicates that a large number of chemical bonds may be buried inside the protein molecule and that the enzyme may have difficulty accessing the active site of zein after pretreatment with ultrasound at a frequency of $68 \mathrm{kHz}$. Notably, the corresponding release of peptides with higher ACE inhibitory activity was more limited with this treatment than with the other working modes and frequencies of ultrasound.

3.4. Conclusion. Ultrasound pretreatment at two frequencies using four working modes was found to significantly improve the angiotensin I-converting enzyme (ACE) inhibitory activity of zein hydrolysates. Zein hydrolysates pretreated with dual sweeping frequency ultrasound at $33 \pm 2$ and $68 \pm$ $2 \mathrm{kHz}$ showed the highest ACE inhibitory activity. The ACE inhibitory activity resulting from single sweeping frequency ultrasound was higher than that resulting from single fixed frequency ultrasound. Dual fixed frequency ultrasound also resulted in a higher ACE inhibitory activity than single fixed frequency ultrasound. However, changes in the degree of hydrolysis (DH) of zein induced by ultrasound were not directly related to the ACE inhibitory activity of zein hydrolysates. Changes in the conformation of zein induced by ultrasound were confirmed by assessment of the solubility, hydrophobicity $\left(H_{0}\right)$, ultraviolet-visible (UV-Vis) spectra, intrinsic fluorescence spectra, and circular dichroism (CD) spectra of zein pretreated by ultrasound, and these changes were consistent with the observed increases in ACE inhibitory activity. Ultrasound pretreatment can significantly improve the enzymolysis properties of proteins; our further studies should help to explain how the different working modes of ultrasound induce protein unfolding and aggregation, influence the enzymolysis properties of proteins, and thus affect the release of bioactive peptides.

\section{Conflicts of Interest}

The authors declare that they have no conflicts of interest.

\section{Acknowledgments}

The authors wish to express their appreciation for the support obtained from the National Natural Science Foundation of China (Grant no. 31501427), China Postdoctoral Science Foundation (Grant no. 2016M601742), the Key Research and Development Program of Jiangsu Province (Grant no. BE2016334), the Foundation of Key Laboratory of Agricultural Products Physical Processing in Jiangsu Province (Grant no. JAPP2014-3), the Senior Professional Research StartUp Fund of Jiangsu University (Grant no. 14JDG180), the Young Backbone Teachers Program of Jiangsu University, the Nature Science Foundation of the Jiangsu Provincial Education Department (Grant no. 11KJB550001), and the Priority Academic Program Development of Jiangsu Higher Education Institutions (PAPD).

\section{References}

[1] C. Zhou, H. Ma, X. Yu, B. Liu, A. E.-G. A. Yagoub, and Z. Pan, "Pretreatment of defatted wheat germ proteins (by-products 
of flour mill industry) using ultrasonic horn and bath reactors: Effect on structure and preparation of ACE-inhibitory peptides," Ultrasonics Sonochemistry, vol. 20, no. 6, pp. 1390-1400, 2013.

[2] X.-X. Li, L.-J. Han, and L.-J. Chen, "In vitro antioxidant activity of protein hydrolysates prepared from corn gluten meal," Journal of the Science of Food and Agriculture, vol. 88, no. 9, pp. 1660-1666, 2008.

[3] M. Juan and J. Carlos, "Zein nanoparticles as vehicles for oral delivery purposes," Nanomedicine, vol. 12, no. 11, pp. 1209-1211, 2017.

[4] J. M. Kim, J. H. Whang, K. M. Kim, J. H. Koh, and H. J. Suh, "Preparation of corn gluten hydrolysate with angiotensin I converting enzyme inhibitory activity and its solubility and moisture sorption," Process Biochemistry, vol. 39, no. 8, pp. 989994, 2004.

[5] S. U. Kadam, B. K. Tiwari, C. lvarez, and C. P. O'donnell, "Ultrasound applications for the extraction, identification and delivery of food proteins and bioactive peptides," Trends in Food Science \& Technology, vol. 46, no. 1, pp. 60-67, 2015.

[6] J. Chandrapala, C. Oliver, S. Kentish, and M. Ashokkumar, "Ultrasonics in food processing," Ultrasonics Sonochemistry, vol. 19, no. 5, pp. 975-983, 2012.

[7] A. C. Soria and M. Villamiel, "Effect of ultrasound on the technological properties and bioactivity of food: a review," Trends in Food Science \& Technology, vol. 21, no. 7, pp. 323-331, 2010.

[8] W. Qu, H. Ma, J. Jia, R. He, L. Luo, and Z. Pan, "Enzymolysis kinetics and activities of ACE inhibitory peptides from wheat germ protein prepared with SFP ultrasound-assisted processing," Ultrasonics Sonochemistry, vol. 19, no. 5, pp. 1021-1026, 2012.

[9] J. Jin, H. Ma, K. Wang et al., "Effects of multi-frequency power ultrasound on the enzymolysis and structural characteristics of corn gluten meal," Ultrasonics Sonochemistry, vol. 24, pp. 55-64, 2015.

[10] Y. Zhang, B. Wang, C. Zhou et al., "Surface topography, nanomechanics and secondary structure of wheat gluten pretreated by alternate dual-frequency ultrasound and the correlation to enzymolysis," Ultrasonics Sonochemistry, vol. 31, pp. 267-275, 2016.

[11] J. Jia, H. Ma, W. Zhao et al., "The use of ultrasound for enzymatic preparation of ACE-inhibitory peptides from wheat germ protein," Food Chemistry, vol. 119, no. 1, pp. 336-342, 2010.

[12] X. Ren, H. Ma, S. Mao, and H. Zhou, "Effects of sweeping frequency ultrasound treatment on enzymatic preparations of ACE-inhibitory peptides from zein," European Food Research and Technology, vol. 238, no. 3, pp. 435-442, 2014.

[13] N. Parris and L. C. Dickey, "Extraction and solubility characteristics of zein proteins from dry-milled corn," Journal of Agricultural and Food Chemistry, vol. 49, no. 8, pp. 3757-3760, 2001.

[14] J. Adler-Nissen, "Enzymic hydrolysis of proteins for increased solubility," Journal of Agricultural and Food Chemistry, vol. 24, no. 6, pp. 1090-1093, 1976.

[15] V. Vermeirssen, J. Van Camp, and W. Verstraete, “Optimisation and validation of an angiotensin-converting enzyme inhibition assay for the screening of bioactive peptides," Journal of Biochemical and Biophysical Methods, vol. 51, no. 1, pp. 75-87, 2002.

[16] J. H. Waterborg, "The Lowry method for protein quantitation," The protein protocols handbook, p. 10, 2009.
[17] A. Kato and S. Nakai, "Hydrophobicity determined by a fluorescence probe method and its correlation with surface properties of proteins.", Biochimica et Biophysica Acta, vol. 624, no. 1, pp. 13-20, 1980.

[18] V. S. Sutkar and P. R. Gogate, "Design aspects of sonochemical reactors: Techniques for understanding cavitational activity distribution and effect of operating parameters," Chemical Engineering Journal, vol. 155, no. 1-2, pp. 26-36, 2009.

[19] R. Feng, Y. Zhao, C. Zhu, and T. J. Mason, "Enhancement of ultrasonic cavitation yield by multi-frequency sonication," Ultrasonics Sonochemistry, vol. 9, no. 5, pp. 231-236, 2002.

[20] P. A. Tatake and A. B. Pandit, "Modelling and experimental investigation into cavity dynamics and cavitational yield: Influence of dual frequency ultrasound sources," Chemical Engineering Science, vol. 57, no. 22-23, pp. 4987-4995, 2002.

[21] P. R. Gogate, S. Mujumdar, and A. B. Pandit, "Sonochemical reactors for waste water treatment: Comparison using formic acid degradation as a model reaction," Advances in Environmental Research, vol. 7, no. 2, pp. 283-299, 2003.

[22] A. Shanmugam, J. Chandrapala, and M. Ashokkumar, "The effect of ultrasound on the physical and functional properties of skim milk," Innovative Food Science and Emerging Technologies, vol. 16, pp. 251-258, 2012.

[23] Q. Huang, L. Lin, and F. Xiong, "Ultrasound effects on the structure and chemical reactivity of cornstarch granules," Starch - Stärke, vol. 59, no. 8, pp. 371-378, 2007.

[24] C. Arzeni, K. Martínez, P. Zema, A. Arias, O. E. Pérez, and A. M. R. Pilosof, "Comparative study of high intensity ultrasound effects on food proteins functionality," Journal of Food Engineering, vol. 108, no. 3, pp. 463-472, 2012.

[25] L. Jiang, J. Wang, Y. Li et al., "Effects of ultrasound on the structure and physical properties of black bean protein isolates," Food Research International, vol. 62, pp. 595-601, 2014.

[26] H. Hu, J. Wu, E. C. Y. Li-Chan et al., "Effects of ultrasound on structural and physical properties of soy protein isolate (SPI) dispersions," Food Hydrocolloids, vol. 30, no. 2, pp. 647-655, 2013.

[27] Y. Sun, J. Chen, S. Zhang et al., "Effect of power ultrasound pretreatment on the physical and functional properties of reconstituted milk protein concentrate," Journal of Food Engineering, vol. 124, pp. 11-18, 2014.

[28] G. R. Grimsley and C. N. Pace, "Spectrophotometric determination of protein concentration," Current protocols in protein science, vol. 3, 2004.

[29] M. I. Viseu, T. I. Carvalho, and S. M. B. Costa, "Conformational Transitions in $\beta$-Lactoglobulin induced by cationic amphiphiles: equilibrium studies," Biophysical Journal, vol. 86, no. 4, pp. 2392-2402, 2004.

[30] S. Li, X. Yang, Y. Zhang et al., "Effects of ultrasound and ultrasound assisted alkaline pretreatments on the enzymolysis and structural characteristics of rice protein," Ultrasonics Sonochemistry, vol. 31, pp. 20-28, 2016.

[31] A. R. Jambrak, T. J. Mason, V. Lelas, Z. Herceg, and I. L. Herceg, "Effect of ultrasound treatment on solubility and foaming properties of whey protein suspensions," Journal of Food Engineering, vol. 86, no. 2, pp. 281-287, 2008.

[32] J. Chandrapala, B. Zisu, M. Palmer, S. Kentish, and M. Ashokkumar, "Effects of ultrasound on the thermal and structural characteristics of proteins in reconstituted whey protein concentrate," Ultrasonics Sonochemistry, vol. 18, no. 5, pp. 951-957, 2011. 

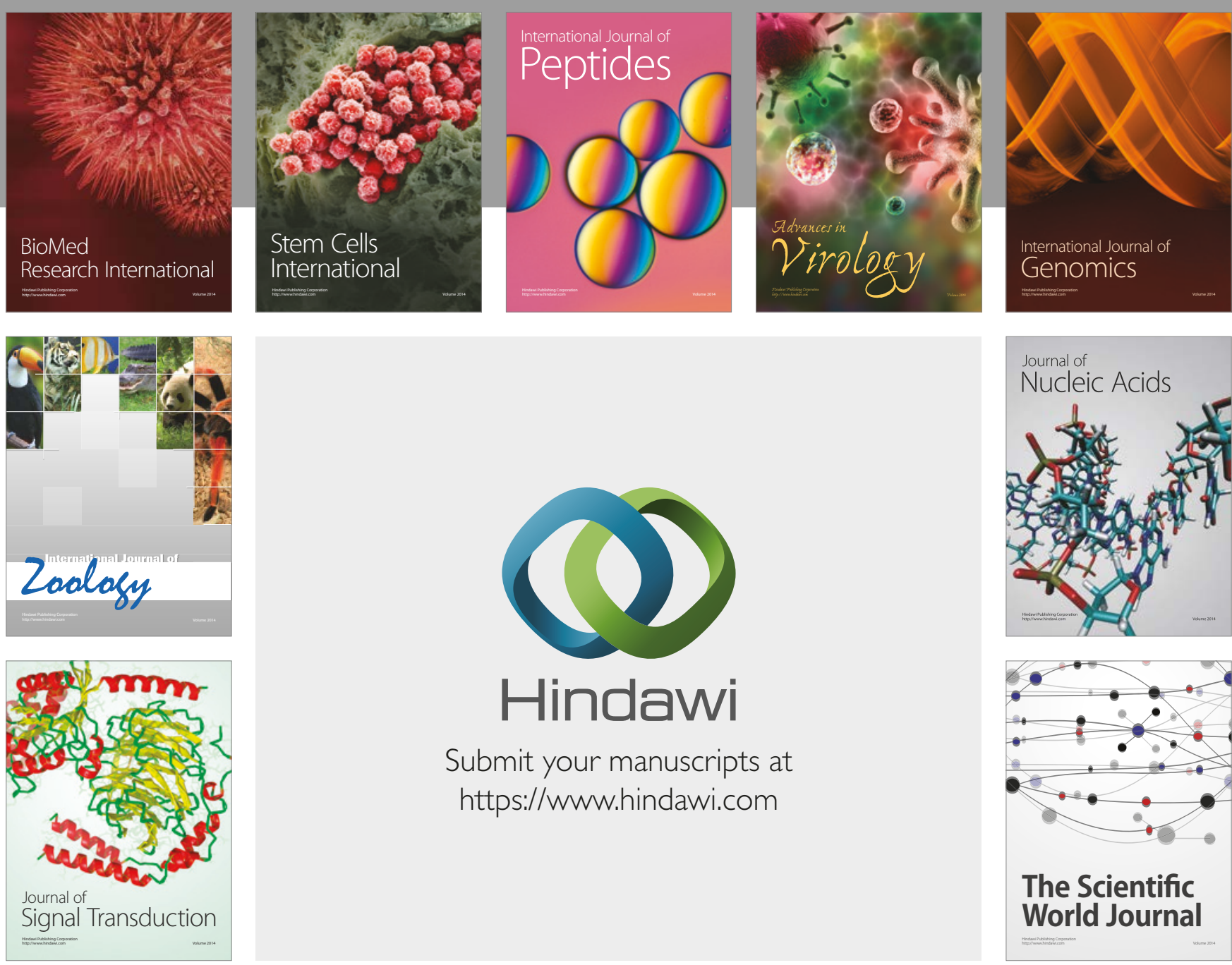

Submit your manuscripts at

https://www.hindawi.com
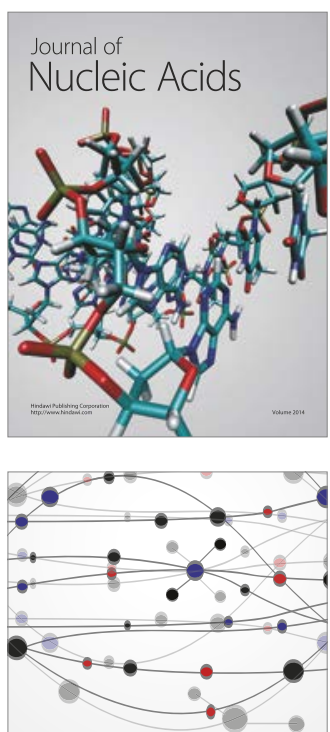

The Scientific World Journal

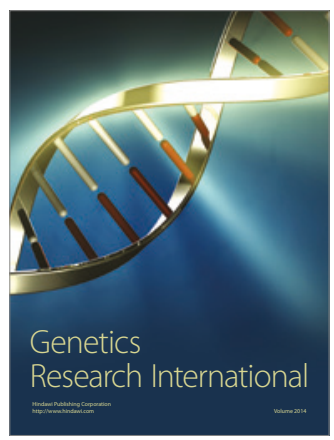

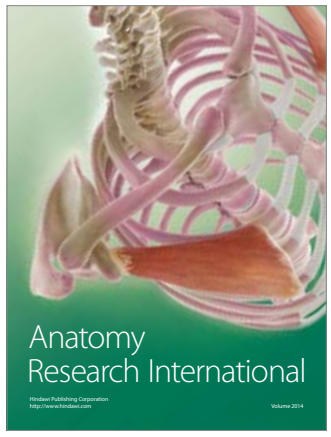

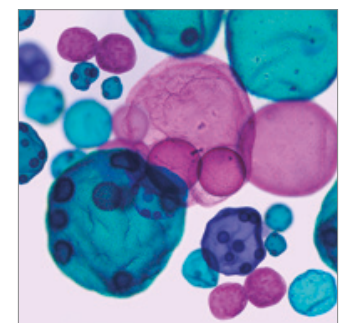

International Journal of Microbiology
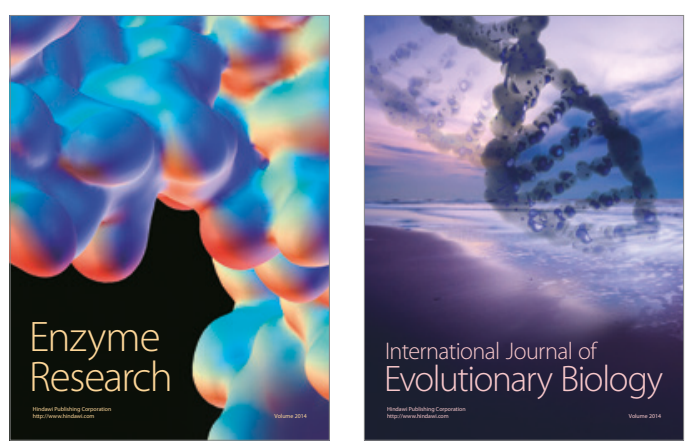
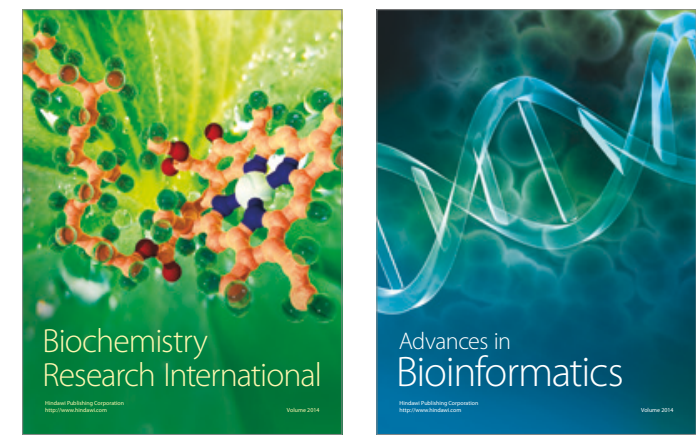

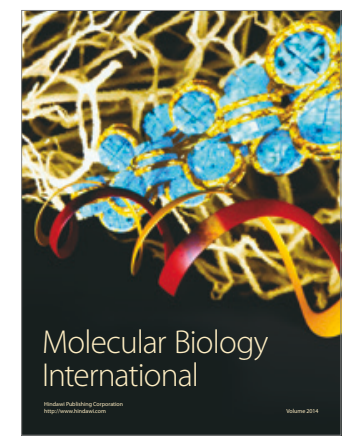

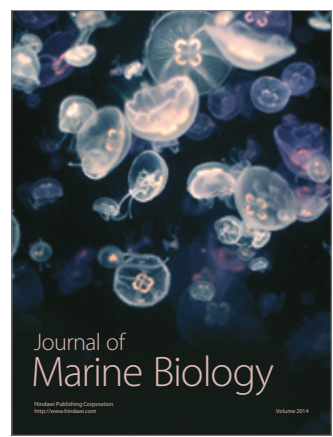

